\title{
On the use of Purcell factors for plasmon antennas
}

\author{
A. Femius Koenderink* \\ Center for Nanophotonics, FOM Institute for Atomic and Molecular Physics (AMOLF), Sciencepark 104, \\ NL-1098XG Amsterdam, The Netherlands. ${ }^{*}$ Corresponding author: f.koenderink@amolf.nl
}

\begin{abstract}
The Purcell factor is the standard figure of merit for spontaneous emission enhancement in microcavities, that has also been proposed to describe emission enhancements for plasmonic resonances. A comparison is made of quality factor, mode volume and Purcell factor for single and coupled plasmon spheres to exact calculations of emission rates. The paper explains why the Purcell factor is not appropriate for plasmon antennas.
\end{abstract}

PACS numbers: 230.5750, 270.5580, 240.6680. Manuscript prepared on July 1, 2010

It is a paradigm of quantum optics that the internal dynamics of a quantum system can be controlled by placing the quantum system in a photonic environment that is resonant with radiative transitions of the source $\stackrel{1}{*}$. Indeed, according to Fermi's Golden Rule, the spontaneous emission decay rate is proportional to the density of photon states that the photonic environment offers for spontaneous decay ${ }^{1}$. A resonant microcavity with a high quality factor $Q$, and a mode confined in an ultrasmall volume $V$ enhances the density of photon states by a factor know as the Purcell factor $Q / V^{1-\underline{3}}$. Creating microcavities with the highest $Q / V$ is a central target in solid state quantum optics, with success being reported with photonic crystals, micropillars, and $\mathrm{SiO}_{2}$ microspheres and microtoroids 2,3 .

Recent advances in nanofabrication have provided new impetus to the field of plasmonics, that creates highly confined electromagnetic resonances by using the response of free electrons in metals ${ }^{4}$. In particular, there are widespread activities that exploit plasmon antennas for enhanced fluorescence and quantum optics with single plasmons ${ }^{4-11}$. Due to absorption and radiative loss, the quality factor of plasmonic devices is extremely low $\left(Q<10^{2}\right)$. Nonetheless, the truly nanoscale confinement leads to estimates for the Purcell factor of order $F=10^{3}$, competitive with dielectric cavities $11 \underline{13}$. Motivated by the tremendous success of the Purcell factor as a quantitative figure of merit for dielectric cavities, several workers have also adopted this figure of merit for plasmonics ${ }^{12-14}$. Here, I demonstrate that the Purcell factor does not provide a quantitative gauge for spontaneous emission enhancements in plasmonic antennas.

The spontaneous emission rate $\Gamma$ of a dipole emitter (transition frequency $\omega$, transition dipole moment $d$ ) is governed by the local density of photonic states (LDOS) $N\left(\omega, \mathbf{r}, \mathbf{e}_{d}\right)$ that enters Fermi's Golden Rule (cf. p. 273-275 in ${ }^{\frac{1}{}}$ ) through $\Gamma\left(\omega, \mathbf{r}, \mathbf{e}_{d}\right)=\frac{\pi d^{2} \omega}{3 \hbar \epsilon_{0}} N\left(\mathbf{r}, \omega, \mathbf{e}_{d}\right)$. Here, $\mathbf{r}$ is the source position and $\mathbf{e}_{d}$ the dipole orientation. In terms of the electric field Green dyadic $\mathbb{G}\left(\mathbf{r}, \mathbf{r}^{\prime}, \omega\right)$ the LDOS equals ${ }^{1,15}$

$$
N\left(\omega, \mathbf{r}, \mathbf{e}_{d}\right)=\frac{6 \omega}{\pi c^{2}}\left(\mathbf{e}_{d}^{T} \cdot \operatorname{Im}(\mathbb{G}(\mathbf{r}, \mathbf{r}, \omega)) \cdot \mathbf{e}_{d}\right) .
$$

This formulation is rigorous even for dissipative plasmonic, systems, in which case Eq. (1) includes quenching 1.8 . The Purcell factor for dielectric cavities rests on two assumptions. First, in non-dissipative systems, normal modes can be defined, and the LDOS (1) can be rewritten as a sum over all the normal modes

$$
N\left(\omega_{0}, \mathbf{r}, \mathbf{e}_{d}\right)=3 \sum_{\lambda, \omega}\left|\mathbf{e}_{d}^{T} \cdot \mathbf{E}_{\lambda}(\mathbf{r}, \omega)\right|^{2} \delta\left(\omega-\omega_{0}\right) .
$$

The second assumption (see Gerard et $\mathrm{al}^{\frac{3}{}}$ ) is that the sum over all modes in Eq. (2) is dominated by just a single term. The $\delta$-function is replaced by a Lorentzian spectrum of width $\Delta \omega=\omega / Q$ centered around the cavity resonance, and the spatial profile is set by the approximate eigenfunction of the cavity, normalized to contain a single photon within its mode volume $V$. Within this approximation, the LDOS $N_{\text {cav }}\left(\omega_{\text {cav }}, \mathbf{r}_{\text {max }}, \mathbf{e}_{d}\right)$ at the spatial and spectral mode maximum, normalized to the LDOS $N_{\text {host }}=n \omega^{2} /\left(3 \pi^{2} c^{3}\right)$ of the host medium (index $n$ ) is well approximated by the Purcell factor

$$
F=\frac{3 Q}{4 \pi^{2} V}\left(\frac{\lambda}{n}\right)^{3} .
$$

Maier has previously noted that reasonable numerical values for $F$ and $V$ can only be obtained for plasmonic systems if the energy density $\epsilon|E|^{2}$ that enters the mode volume $V=\int \epsilon|E|^{2} d \mathbf{r} / \max \left(\epsilon|E|^{2}\right)$ is redefined ${ }^{13}$ to avoid, e.g., negative energy densities 13,16 . The proper energy density that is positive and real even when $\operatorname{Re}(\epsilon)<0$, and that accounts for energy stored in the material was derived by Rup$\operatorname{pin}^{13,16}$. Following Ruppins formalism ${ }^{16}$, the energy density used here is $(\operatorname{Re} \epsilon+2 \omega \operatorname{Im} \epsilon / \gamma)|E|^{2}$, appropriate for a Drude model $\epsilon=1-\omega_{p}^{2} / \omega(\omega+i \gamma)$ for the dielectric constant of silver $\left(\omega_{p}=7.9 \mathrm{eV}\right.$, damping rate $\left.\gamma=0.06 \mathrm{eV}\right)$.

In this work, the commonly employed procedure to obtain $F$ described above is compared with exact calculations of the LDOS for the textbook case of metal spheres. First, I consider calculation of the mode volume. For dielectric cavities, one typically runs a numerical solver to obtain the local field. As long as the excitation has spatial and spectral overlap with the cavity mode, a high $F$ ensures that the calculated spatial distribution of the local field of the resonance is almost independent of the exact excitation. The field distribution (total field minus excitation) is used to evaluate the mode volume integral, with integration limits truncated at the computation domain. Also for plasmon antennas, far field excitation yields near-field patterns with large local field enhancements, representative of the resonance mode profile. In this work, the exact Green function for a sphere $\frac{15}{}$ is used to calculate the local energy density using plane wave excitation. Figure 1 a) shows a 
(a)

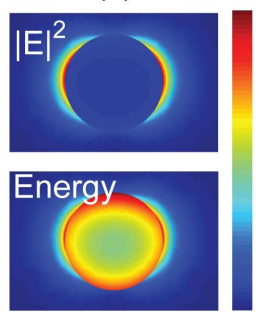

(c)

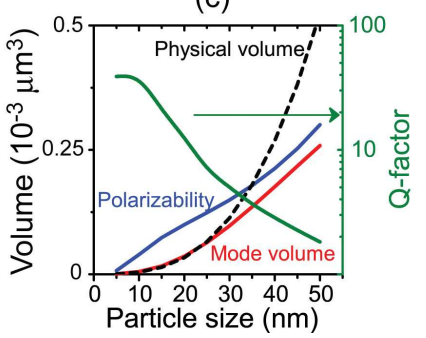

(b)

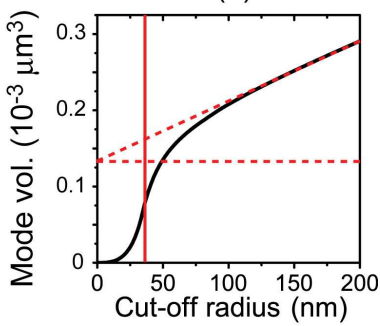

(d)

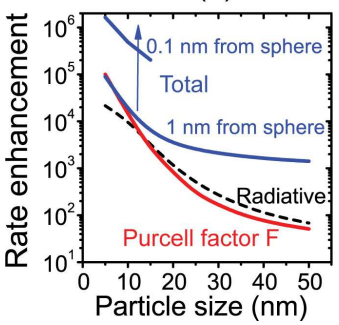

FIG. 1: (Color online) Contour plots of electric field $|E|^{2}$ and energy density $W$ for a $35 \mathrm{~nm}$ radius silver sphere in vacuum (plane wave excitation from below, horizontal polarization). The colorbar ranges from 0 to 107 in units of the incident $|E|^{2}$, resp. 0 to 118 in units of $W$ of the incident wave. (b) Mode volume $\int W d \mathbf{r} / \max \left(\epsilon(r)|E|^{2}\right)$ evaluated over a spherical integration domain truncated at $R$, plotted versus $R$. Vertical line: particle radius. Dashed line: linear divergence due to radiation loss. Horizontal line: mode volume. (c) Mode volume (red), polarizability (blue) and physical volume (dashed) versus sphere radius for $\mathrm{Ag}$ spheres in vacuum. The right hand scale refers to the quality factor $Q$ (green). (d) Red curve: Purcell enhancement calculated from Q and V. Dashed: exact radiative decay rate. Solid curves: total decay rate for a dipole at $1 \mathrm{~nm}$ and $0.1 \mathrm{~nm}$ from the sphere.

strongly enhanced and confined field (enhancement relative to incident $|E|^{2}$ by $\sim 10^{2}$ ) just outside the sphere (sphere radius $35 \mathrm{~nm}$ ). The energy density reaches a maximum just inside the sphere. In Figure 1 b) a plot is shown of the mode volume integral evaluated over a truncated integration domain of radius $R$ around the plasmon sphere. Figure 1 (b) exemplifies a hitherto unnoted problem that is generic for all radiatively damped resonances $\frac{17}{-}$, including all plasmon antennas. The mode volume integral shows a linear divergence with the truncation radius $R$. The divergence is understood by noting that radiative loss signifies constant integrated far-field flux, and hence a $1 / R^{2}$ asymptotic fall-off of $|E|^{2}$. For dielectric cavities, this divergence is generally not apparent as high Q's imply a very small slope of the linear tail in Fig. 1 b), that is often negligible in a finite numerical computation domain. Leaving the fundamental treatment aside ${ }^{17}$, I note that for dielectric cavities a rigorous mode volume can be retrieved by subtracting the radiative part (linear divergence). Figure 1 (c) shows the divergence-corrected mode volume for silver spheres of different sizes. The mode volume is generally comparable to, or even smaller (large spheres) than the physical volume, since the field energy is mainly stored near the metal surface.

To obtain the quality factor $Q$, Mie extinction cross sec-

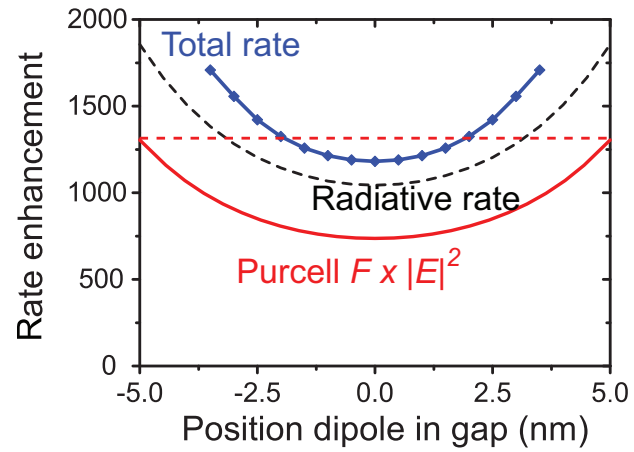

FIG. 2: Radiative decay rate (dashed) and total decay rate (joined symbols) enhancement along the centerline joining two $25 \mathrm{~nm}$ silver spheres with a $10 \mathrm{~nm}$ gap. Enhancement is relative to the rate in the $n=1.5$ host medium. Red: prediction from Purcell theory. The dipole orientation is along the centerline. Right: contour plot of $|E|^{2}$ (scale from 0 to 1890 in units of incident $|E|^{2}$, dimer illuminated by a vertically polarized plane wave).

tions $s^{15}$ are analyzed. By dividing out the non-resonant $\omega^{4}$ scaling, Lorentzian line shapes are retrieved from which $Q$ can be extracted. Figure 1(c) shows that the quality factor decreases from $Q \sim 40$ (limited by absorption) in very small particles to $Q \sim 3$ for larger particles. Combining $Q$ and $V$ in Fig. 1(d) reveals predicted Purcell factors that range from $10^{5}$ for the smallest spheres to below $10^{2}$ for the $50 \mathrm{~nm}$ spheres. The key question is in how far this Purcell factor $F$ correctly predicts the emission rate enhancement calculated rigorously from $\operatorname{Im} \mathbb{G}^{15}$. For this comparison, the maximum total decay rate enhancement almost at the particle surface (separations shown: $1 \mathrm{~nm}$ and $0.1 \mathrm{~nm}$ ), where the mode field is maximum, is plotted in Fig.1(d). The Purcell factor clearly underestimates the total radiative decay rate by an order of magnitude. Strictly speaking the, Purcell factor is an estimate for the LDOS, i.e. the total decay rate. The Purcell factor estimates the radiative decay reasonably for particles $>15 \mathrm{~nm}$ radius.

One might argue that single plasmon spheres set a poor example, as they do not provide very high local field enhancements typical for plasmonics. Figure 2 presents an analysis of the Purcell factor for two spheres with a narrow gap, with a highly confined gap mode. The calculation uses a multipole expansion multiple scattering code provided by García de Abajo $\frac{18}{2}$ to analyze a dimer of two $25 \mathrm{~nm}$ radius silver spheres separated by a $10 \mathrm{~nm}$ gap, in a $n=1.5$ host medium. A scattering resonance with $Q=5.7$ occurs near $\lambda=506 \mathrm{~nm}$, with a 1890 times $|E|^{2}$ enhancement polarized along the dimer axis. Purcell analysis predicts a mode volume $V=1.32 \cdot 10^{-5} \mu \mathrm{m}^{3}$ and a rate enhancement ranging from $F \sim 1300$ at a sphere surface to about 700 times exactly in between the spheres. The rigorous calculation predicts a rate enhancement of $\sim 1200$ times exactly in the middle of the gap. Again the Purcell factor underestimates the actual total and radiative decay rate enhancements by almost a factor of 2 .

Referring to the two assumptions underlying the Purcell factor, in general both assumptions will break down: Firstly, a set of normal modes can generally not be defined for dispersive absorbing media. Secondly, if an expansion in modes can 
be generalized (as in the case of multipole expansion for the Green function of a plasmon sphere) no single term is dominant, even if the resonance is clearly due to one term. For instance, at the dipolar plasmon sphere resonance, the LDOS is dominated by non-resonant coupling of the emission dipole to higher order multipole moments of the plasmon sphere ${ }^{8,20}$. This nonresonant coupling is not contained in a Purcell factor estimate based on $Q$ and $V$, and corresponds to immediate quenching of emission when very close to the metal. The total decay rate diverges as $z^{-3}$, where $z$ is the separation between dipole and metal surface $\frac{19,20}{}$. Such quenching occurs generally in both planar and curved plasmonic structures ${ }^{20}$, and unfortunately always exactly coincides with the field maximum where one seeks to apply the Purcell factor. In the single sphere case, the fact that the Purcell factor reasonably approximates the radiative rate is consistent with the fact that most non-resonant coupling is quenching. For the dimer case, if the dipole is in the middle of the gap, multipole contributions enhance both the total and radiative decay rate while maintaining a high quantum efficiency.

Based on the above, one is led to conclude that the Purcell factor is not adequate for plasmon antennas. One might expect that moving the emitter outside the regime of the extreme near field limit allows one to retrieve the Purcell enhancement prediction, taking into account that displacement and detuning of the emitter causes the enhancement to decrease from
$F$ in proportion to the mode field and the Lorentzian cavity lineshape ${ }^{1,3}$. Even then there is a fundamental difference that sets plasmon antennas aside. Contrary to Purcell theory, the emission rate of a dipole near a nano-antenna can follow a dispersive Fano-type frequency dependence ${ }^{8,22}$. As first discussed by Dulkeith et al. 22 the lineshape can be understood as destructive interference of radiation emitted by the emission dipole with that radiated by the plasmon antenna. This phenomenon occurs for all antennas where coupling to radiation is important. Incidentally, in this regime the decay enhancement can be well understood in a point dipole model ${ }^{21}$. The only relevant volume that quantifies the interaction in this case is the polarizability (Fig. 11), not the mode volume.

In closing, the Purcell factor appears to be unsuited as a general gauge to classify the emission enhancements of plasmonic resonances quantitatively. While predicted Purcell factors may act as a qualitative design guideline, in practice one always needs to confirm by a full calculation that non-resonant channels are negligible.

I thank Javier García de Abajo for source code, and Ad Lagendijk and Mischa Bonn for discussion. This work is part of the research program of the "Stichting voor Fundamenteel Onderzoek der Materie (FOM)," which is financially supported by the "Nederlandse Organisatie voor Wetenschappelijk Onderzoek (NWO)." This research is further supported by a NWO VIDI fellowship.
${ }^{1}$ L. Novotny and B. Hecht, Principles of Nano-Optics (Cambridge University Press, Cambridge, 2006).

${ }^{2}$ K. J. Vahala, ed., Optical Microcavities (World Scientific, Singapore, 2004).

3 J.-M. Gérard and B. Gayral, Purcell effect for InAs quantum boxes in three-dimensional solid-state microcavities, J. Lightwave Technol. 17, 2089 (1999).

4 J. A. Schuller, E. S. Barnard, W. S. Cai, Y. C. Yun, J. S. White, and M. L. Brongersma, Plasmonics for extreme light concentration and manipulation, Nature Mat. 9, 193 (2010).

5 P. Anger, P. Bharadwaj, and L. Novotny, Enhancement and quenching of single-molecule fluorescence, Phys. Rev. Lett. 96, 113002 (2006).

6 S. Kühn, U. Håkanson, L. Rogobete, and V. Sandoghdar, Enhancement of single molecule fluorescence using a gold nanoparticle as an optical nano-antenna, Phys. Rev. Lett. 97, 017402 (2006).

7 O.L. Muskens, V. Giannini, J.A. Sánchez Gil, and J. Gómez Rivas, Strong enhancement of the radiative decay rate of emitters by single plasmonic nanoantennas, Nano Lett. 7, 2871 (2007).

${ }^{8}$ H. Mertens, A. F. Koenderink and A. Polman, Optimizing plasmon-enhanced luminescence near noble-metal nanospheres: comparison of the exact model and the Gersten and Nitzan approach, Phys. Rev. B 76, 115123 (2007).

9 T. H. Taminiau, F. D. Stefani, F. B. Segerink and N. F. van Hulst, Optical antennas direct single-molecule emission, Nature Photon. 2234 (2008).

10 A. Kinkhabwala, Z. Yu, S. Fan, Y. Avlavesich, K. Müllen, and W. E. Moerner, Large single-molecule fluorescence enhancements produced by a bowtie nanoantenna, Nature Photon. 3, 654 (2009).

11 A. V. Akimov, A. Mukherjee, C. L. Yu, D. E. Chang, A. S. Zibrov,
P. R. Hemmer, H. Park, M. D. Lukin, Generation of Single Optical Plasmons in Metallic Nanowires Coupled to Quantum Dots, Nature 450, 402 (2007).

12 M. Kuttge, F.J. García de Abajo and A. Polman, Ultrasmall Mode Volume Plasmonic Nanodisk Resonators, Nano Lett. 10, 1537 (2010).

13 S. A. Maier, Plasmonic field enhancement and SERS in the effective mode volume picture, Opt. Express 14, 1957 (2007).

14 R. F. Oulton, G. Bartal, D. F. P. Pile and X. Zhang, Confinement and propagation characteristics of subwavelength plasmonic modes, New. J. Phys. 10, 105018 (2008).

15 C.-T. Tai, Dyadic Green Functions in Electromagnetic Theory, 2nd ed. (IEEE, New York, 1993).

16 R. Ruppin, Electromagnetic energy density in a dispersive and absorptive, Phys. Lett. A 299, 309 (2002).

17 P. de Vries, A. F. Koenderink and A. Lagendijk, to be published.

18 F. J. García de Abajo, Multiple scattering of radiation in clusters of dielectrics, Phys. Rev. B. 60, 6086 (1999).

19 G. W. Ford and W. H. Weber, Phys. Rep. Electromagnetic interactions of molecules with metal surfaces, 113, 195 (1984).

20 E. Castanié, M. Boffety, and R. Carminati, Fluorescence quenching by a metal nanoparticle in the extreme near-field regime, Opt. Lett. 35, 291 (2010)

${ }^{21}$ P. de Vries, D.V. van Coevorden, and A. Lagendijk, Point scatterers for classical waves, Rev. Mod. Phys. 70, 447 (1998).

${ }^{22}$ E. Dulkeith, A. C. Morteani, T. Niedereichholz, T. A. Klar, J. Feldmann, S. A. Levi, F. C. J. M. van Veggel, D. N. Reinhoudt, M. Möller, and D. I. Gittins, Fluorescence Quenching of Dye Molecules near Gold Nanoparticles: Radiative and Nonradiative Effects, Phys. Rev. Lett. 89, 203002 (2002). 